\title{
Assistance for Learning Design Community A context-awareness and pattern-based approach
}

\author{
Jean-Pierre Clayer ${ }^{1}$, Claudine Piau-Toffolon ${ }^{1}$ and Christophe Choquet $^{1}$ \\ ${ }^{I}$ LIUM (computer laboratory),University of Maine, PRES UNAM, Avenue Olivier Messiaen, Le Mans, France \\ \{jean-pierre.clayer, claudine.piau-toffolon, christophe.choquet\} @ univ-lemans.fr
}

Keywords: $\quad$ Learning Design, Design process, Assistance, Context-Awareness, Patterns, Pedagogical Design Scheme

\begin{abstract}
Designing learning is a complex task. Researchers and teachers have proposed many methodological issues to deal with it. Despite valuable technological advantages of machine-readable outcomes, most of the time these proposals are too complex to use, limiting the expressiveness, the sharing and reuse of learning scenario by the teachers' community. Learning design processes support and guidance are often missing or insufficiently adaptable to the design context. Based on the needs of an association dealing with professional integration in charge with back-to-work programs named PARTAGE, we defined a design approach based on patterns and an editing tool to support the learning design activities to help the sharing of pedagogical scenarios inside the teachers' community of PARTAGE. In this work, we focus our attention on the assistance abilities of the editing tool, depending of its design context awareness. We realize a pilot study on this approach according to a participatory design approach with trainers of the association and the research team in charge with the study.
\end{abstract}

\section{INTRODUCTION}

According to Laurillard, teaching may be considered as a design science (Laurillard, 2012, p1). Learning Design is a complex task (Rohse and Anderson, 2006). The learning design research field produced a set of design process, methods and associated tools such as Instructional design models, the MISA Instructional Engineering Method (Paquette, 2004), learning design specifications like PALO, LDL or the IMS-LD learner activity-driven approach (Koper \& Olivier, 2004).

Researchers in Technology Enhanced Learning (TEL) get increasing interest within approaches based on patterns. Patterns are potentially valuable tools for designing complex learning management systems. Design approaches based on patterns introduce a way to negotiate the new emergent roles of teachers and learners by, for instance, allowing the learner to assume a designer role, based on the instantiation (contextualization) of a pattern on a given learning situation (Rohse and Anserson, 2006). By example, learners or teachers may assume a designer role criticizing or refining a pattern solution as the context evolves on a learning situation. These approaches are immediately relevant to teachers as they present means by which the teaching community can participate in design. The knowledge and the know-how developed by the teacher is externalized, embodied in patterns sharable in a community that can critique and build its knowledge (Laurillard, 2012, p8). COLLAGE, a collaborative learning flow pattern (CLFP) editor (Hernandez Leo \& al, 2006) proposes a visual pattern based design approach implementing learning scenarios in RELOAD. This approach is based on the IMS LD specification which enables the modeling of learning processes. The MDEduc project proposes a Pedagogical Patterns Editor for the design of learning scenario. This is a text editor using the formalism and the syntax of patterns. ScenEdit and the ISIS model support also a pattern based approach to design learning scenarios. Teachers can share and reuse practices with patterns as far as they could be expressed with a pedagogical oriented vocabulary and syntax.

Nevertheless, these approaches propose teachers to identify the best solution (pattern) among existing ones for their pedagogical needs according to a given pedagogical model, reflecting 
a specific pedagogical approach. We've named such models pedagogical design schemes (PDS).

Nevertheless, these approaches have some limitations. One of them is the lack of design assistance mechanisms (Villiot, 2007). According to (Dufresne \& al, 2003), two assistance levels could be defined : the first level is to provide users with an editing tool for designing a pedagogical scenario, the second one is to offer a support for design activity with such an editor. In a design context where instantiating patterns are emphasized, we introduce a third level of assistance, for helping the users to reuse existing patterns and to share new ones.

Thus, our main issue is to define a design approach based on the instantiation of shared patterns, by using an editing tool where the design activity benefits from contextualized assistance which takes into account user own design approach identified as pedagogical design scheme.

Our technical approach is in the scoop of Domain Specific Modeling. It allows designers (assisted by modeling experts if needed) to define their own Educational Modeling Language by specifying a domain-specific language and to use it for building their scenarios (El Kechaï \& Choquet 2006). This approach enables the design of computational models (i.e. models interpretable by a computer) which could be enacted by compliant systems.

The work we relate here was primarily based on the needs expressed by PARTAGE, an association dealing with professional integration in charge with back-to-work programs. Initially, the persons in charge of this association wanted to produce computerized pedagogical materials in order to facilitate capitalization, reuse and adaptation. Then, they have stressed the need of a tool to support their design sessions in order to produce scenarios more adapted to their heterogeneous public. Finally, considering the important turnover of their training staff, they wanted to capitalize the training know-how of experimented teachers, to be able to share it with novices ones and to provide assistance when trainers are involved in the design of a learning scenario. We worked with trainers of this association within an iterative participatory design methodology, in order to fit better with their needs. The results of this pilot study consists in (1) a design approach based on patterns (Clayer \& al, 2013), (2) an editing tool which allows different pedagogical design scheme models, (3) a way of sharing pedagogical scenarios in a community or, more exactly, patterns which could be instantiated by any member of a community, for his/her own design, and (4) an assistance system that guides teachers during the learning design activity, according to their pedagogical design scheme.

In this paper, we focus on the characterization of the assistance system we have developed for PARTAGE (section 3) and we relate the first experimental uses of the editor by trainers of the association (section 4) in a pilot study. We conclude this paper by some considerations on the possibility to generalize the assistance approach we have defined (section 5). The next section of this paper presents the relevant notions of patterns, user assistance, context-awareness and pedagogical design schemes.

\section{ASSISTANCE FOR LEARNING DESIGN}

\subsection{Patterns}

A pattern is a semi-structured description of an expert's method for solving a recurrent problem which includes a description of the problem itself and the context in which the method is suitable (Mor \& Winters, 2007). Patterns are "good solutions" to deal with complexity characterizing the educational field (Rohse and Anderson, 2006). This formalism offers the teacher an opportunity to externalize his knowledge (Goodyear, 2005) and can be formally expressed. A pattern is defined by three properties: a problem, the context of this problem and a solution. Each pattern captures the best way to solve a problem in a particular context. Patterns guide rather than prescribe (Rohse and Anderson, 2006). A pattern language describes the relations between patterns (associated, composed of...) that capture the whole design process and can guide the designer through step-by-step design guidelines.

According to the Domain Specific Model approach we have adopted for this research work, a meta-model of patterns has been defined to describe the pattern language and a set of patterns has been elaborated and presented in (Clayer \& al, 2013).

\subsection{User assistance}

(Rech \& al, 2007) defines several dimensions for an assistance system:

- the presentation of assistance to the user;

- the way to define assistance algorithms.

Each assistance system could reify the assistance by different kinds of actions: 
- The adaptation (Andresen \& Gronau, 2005) is defined as a characteristic for a system to identify the capacity of this system to modify its structure according to situations or events.

- $\quad$ The retro action (Dufresne \& al, 2003) improves the activity by highlighting or focusing on the elements to complete.

- The advice suggests process or task to realize in order to reach user's goals.

- The constraint ensures consistency with the element affected by this constraint.

The user's task assistance we present in this paper implements these four kinds of actions, with a particular focus on the reuse of the pedagogical elements and scenarios.

\subsection{Context awareness}

Context-aware systems (Bardram, 2005) are well-suited to support adequately the learning design activity of teachers and trainers. An assistance system uses context to provide information and/or services to the user, where relevancy depends on the user's task (Dey \& Abowd, 2000). User's task refers to the interaction between the system and the user during the design session.

Thus, we have proposed a learning design adaptive process based on the user's design context and the design activity. It addresses an automatic user modeling approach where the user's model is defined during a tool design activity session from user's characteristics (user profile, design session's context, and user skills) and its interaction with the system.

User models and user modeling are key elements for personalizing interaction. User modeling is motivated by differences in individual user's needs and characteristics and heterogeneity between different groups of people (Razmerita \& al, 2003). User's characteristics and interactions between the user and the system are relevant tracks to collect and will be used to help teachers/trainers to manage their learning design activity.

\subsection{Pedagogical Design Schemes}

The characteristics of a learning scenario are mainly conditioned by the choice of the pedagogical approach and the learning strategy (Paquette, 2004). Commonly, communities of teachers could be identified by the same context of teaching: a class level, a learning domain, a pedagogical approach. More rarely is, for a community of teachers or trainers, the sharing of a particular pedagogical design scheme (PDS).
A pedagogical design scheme is the approach for designing a course, in our context, a learning scenario. A pedagogical design scheme is defined by an oriented graph of the pedagogical elements (e.g., in the context of IMS-LD, a role, an activity, a learning objective, etc.). The first pedagogical element defined by the designer is the root of the oriented graph and gives the orientation of the PDS. When practicing teachers design a learning scenario with the same design objective, the pedagogical design schemes could be different. For the same design problem, many pedagogical design schemes could exist. Some practitioners, depending on their experience or on the context of design, could start a design by defining resources of the scenario and then, in a second time, related activities and strategies. Some other designers will begin by describing objectives and intentions of the scenario first and associated activities and resources afterwards.

Early instructional design approaches have developed concepts for systematically designing instructional materials. The IMSL-LD specifications are based on a learner activity driven approach (Koper \& Olivier, 2004). This kind of languages provides best practice guides driven by the efficiency of the modeling rather than pedagogical design schemes. Editors developed for these languages want usually to instrument the modeling activity guided by a given design approach, underlying implicitly the interfaces. Some authors identified pedagogical design schemes driven by strategies and intentions. This kind of schemes supports the design process in the ISIS model (Emin, 2010).

We think that, for tooling and supporting a design community of learning scenarios, one has to develop designs tools allowing different pedagogical designs schemes. Thus, we have developed such an editor and performed a pilot study with trainers of a same community of teaching for evaluating its usability.

\section{ASSISTANCE IN DESIGN APPROACH}

User designs pedagogical scenarios composed of pedagogical elements (activity, learning strategy, objective, role, material resource, learning situation, etc.) represented by patterns in our learning design approach. A simplified graphical description of a process pattern for the activity "Realize an individual evaluation/training" is proposed (Fig 1). 


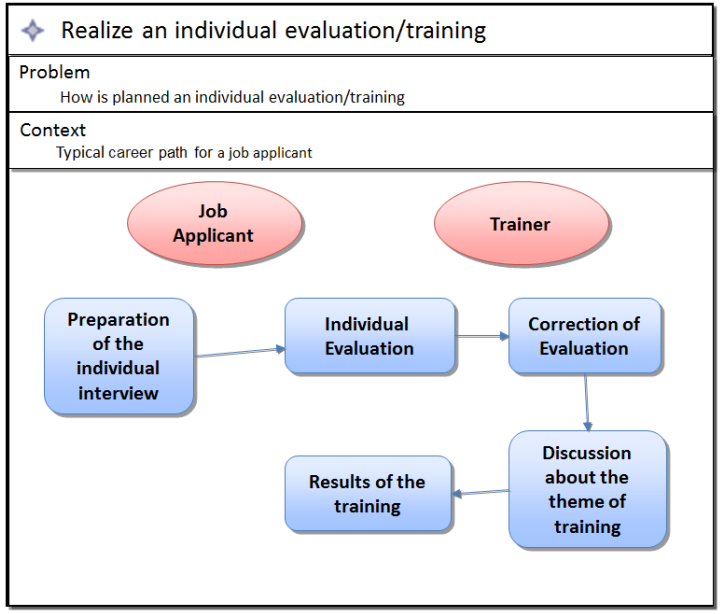

Figure 1: Simplified Graphical Form of a Pattern

To support the user during his design activity, we define (1) a "designer context", e.g. a data structure for capitalizing the information on the designer's pedagogical practices and actions, (2) a task model to identify the current design activity in accordance with the pedagogical design scheme of the designer, and (3) an assistance process which performs a set of assistance actions adapted to the designer context and the current activity.

\subsection{Designer Context}

A "context" is a structured set of information that can be used to characterize the situation of an entity (a person, place or object) that is considered as relevant to the interaction between a user and an application, including the user and the application themselves (Dey \& Abowd, 2000).

In our approach, we refer to the context of a designer to qualify the context of a person, a user (a teacher or trainer) in his learning design activity. This designer context may be characterized by three facets:

- The User Profile (Amato \& Staraccia, 1999) is defined with the information based on the interaction with the system, user preferences and user knowledge. Examples of these data are: the level of adaptation expected in the tool (automatically, with the confirmation of the user, minimum adaptation), the login and password used to identify the user, etc. All of these data could be changed at any time by the designer.

- The Design session's context is the context created during one design session. Each designer context saves the review of all design session's contexts (one by effective session, and by user). This context is composed of :

- The Pedagogical context qualifies learning strategies and objectives defined by the designer.

- The Institutional context is a set of information on the constraints, rules and resources imposed or available to the designer by his/her institution (in this paper, the association PARTAGE).

- The Domain gathers, by the help of keywords, the information concerning the concepts of the user learning domain.

- The Context of use gathers information during the designing session in order to qualify the nature of the task: the Pedagogical Design Scheme and Assistance actions.

- The User's Skills are the skills the designer is supposed by the system to master, as far as he/she used the functionality of the tool (for example: "to reuse a pedagogical element" or "to link a role and an activity together").

User Profile is a static set of information filled by a form during the first session and/or actualized on designer's demand. Design session's context is updated by the assistance process during a design session. When a user creates a resource, this resource is capitalized in the Institutional context as a resource available inside the relative institution. User's Skills is the part of the context taking into account the user experience. It is updated depending the nature of the interaction and the user's actions.

\subsection{Task model of design}

Task models are useful for supporting the user during a session. With such a task model and by defining links between tasks and the interaction devices composing the user interface, an editor could provide context-sensitive, task-oriented support to the designer (Pangoli \& Paternò, 1995). Considering our objective, we have chosen to adapt the user task model defined in (Paternò, 2001), as it is centered on the task of the user.

We have defined two levels in the task model:

- The first level is the pedagogical design scheme itself. The pedagogical design scheme is updated after each task performed by the user.

- The second level is a tree of tasks. The tree of tasks is associated with a category of 
pedagogical elements and represents the tasks that the user is able to perform during a design session with these pedagogical elements (Fig.2).

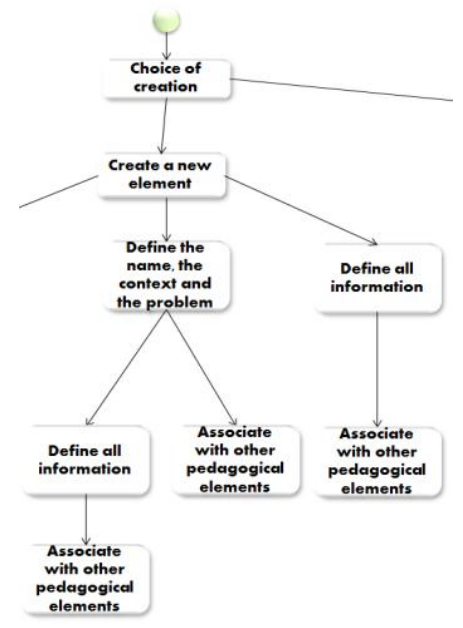

Figure 2: Partial Task-Model for the creation of an element.

\subsection{Assistance actions}

As described above, the assistance can lead on many kinds of actions (message of the system, guidance, adaptation...) (Dufresne \& al, 2003). We have implemented four kinds of assistance actions in the assistance system:

- Guiding: The system supports the user with a guide of practices, adapted to the current design activity. The textual information relative to the current action is highlighted and changes according to the next user's action. This assistance should enable the appropriation by the designer of the best practices of design regarding its Pedagogical Design Scheme.

- Consistency ensuring: The system informs the user if the current user's action has an impact on the coherence of the scenario. For example, if an activity is defined with a given resource, and if this resource is already registered in the system, the system informs the user that the resource exists and it can be added automatically to the current design model. This assistance ensures that the pedagogical scenario is consistent (by checking on the proprieties of elements, the meaning of combinations or associations of elements) at the end of the design session.
- Action anticipating: The system anticipates the next design action on the basis of the model-task, the pedagogical design scheme and the current design activity. Firstly, the pedagogical design scheme is used to identify the type of pedagogical element currently handled by the designer; then the task model associated to this pedagogical element is used to identify the current design activity. For example, if the user has created many activities, added a material resource and associated this resource to an activity according to its definition, the system verifies and creates all resources required by an activity (referenced in the definition of an activity).

Retroaction providing: The system uses the pedagogical design scheme and the current design activity to verify and modify each pedagogical elements of the current design activity. For example, if the user changes the prerequisites of an activity, then the system has to change the associated pedagogical elements.

The assistance actions are implemented by a rule-based system. A set of rules is defined for each design activity and inferred among a forward chaining, fired by user/system interaction events.

\subsection{Assistance process}

We have defined the assistance process as an iterative cycle of four steps, as the one synthesized in (Lopisteguy \& al, 2012).

- Step 1 "Collect information". The system collects information: the pedagogical design scheme of the session as well as the information describing the current user action and the designer context.

- Step 2 "Qualification". The system identifies as the current design activity, the last activity started in the pedagogical design scheme. The context of the designer is updated with the information collected.

- Step 3 "Diagnostic". The system searches in task-model the current activity and selects the set of rules associated. This set of rules is composed by rules for each type of assistance actions. The rules are contextualized (variables are valued with the collected information). Then, the current design session's context is checked in order to ensure the consistency. By analyzing the task model, the system selects the actions to anticipate. The guide is selected or updated regarding the 
current activity. The set of design session's contexts is used to provide retroactions. The user's skills and the level of assistance are updated in the user profile and used to fix the level of selected assistance actions. Finally, the system elicits the action(s) associated to the best contextualized rule(s) to be applied.

- Step 4 "Adjustment". The system applies the qualified assistance actions and, if necessary, informs the designer of the assistance applied (example of message: "A new resource has been added to the scenario").

This assistance process is active along the whole design process (Clayer \& al, 2013). We will highlight this process in the next section, with a first test out of an editor implementing this assistance process.

\section{PILOT STUDY}

\subsection{Description of the pilot study}

We realize a pilot study with the professional trainers of PARTAGE. Three main characteristics concern this association:

- PARTAGE provides trainings essentially based on formative evaluations supported by classical pedagogical resources and methods (teacher-learner based strategy).

- Pedagogical resources are not always welladapted to the public. Learners are adults unused to be trained and being at a loss with classical paper-based aid. Moreover, they often have difficulties with the basics knowledge of reading and writing or oral expression.

- The association faces many changes within its trainers staff. This turnover doesn't facilitate the reuse and the sharing of the teaching know-how.

This pilot study takes place in a design participatory approach (Schuler, A Namioka, 1993). Ten trainers of the association took part in this pilot study. The pilot study has been conducted during three iterations which have each occurred during a period of two or three weeks the last two years.

In a first step, after having observed and analyzed the practice of the association, we have proposed some patterns (Clayer \& al, 2013) coconstructed with the trainers.

In a second step, during one of these sessions of participatory design, we have assigned the trainers with a new learning situation to design.
Each of them has designed a complete scenario of the learning situation, creating by the way many pedagogical elements. This was the opportunity for us to identify and then to model different Pedagogical Design Schemes.

We have then developed an editor, taking into account the PDS and the patterns previously formalized, and implementing the assistance process we have defined.

\subsection{An editing tool}

The editor is generated into the EMF-GMF framework (Steinberg \& al, 2008). EMF-GMF framework supports fast update cycles of development for tools (Krogmann \& Becker, 2007). The interface of the tool allows the designer to visualize the pedagogical elements he/she instantiates in a graphical view of the solution (Fig. 3-A). A toolbox provides the design primitives (pedagogical elements: objective, pedagogical strategy, material resource, human resource, activity, learning situation) (Fig. 3-B). Finally, a visual description for the information concerning the pedagogical element is accessible through the tab property (Fig. 3-C).

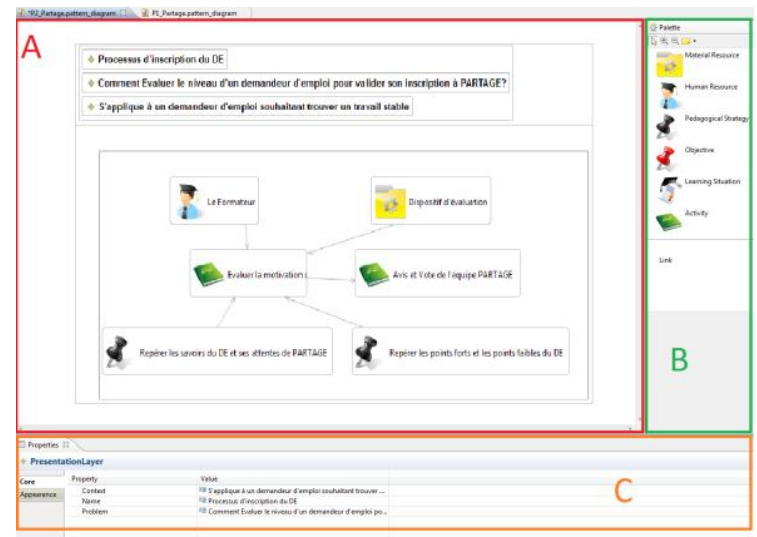

Figure 3: The interface of editing tool.

\subsection{Test out of the assistance}

In a third step we have proposed to two trainers to test out the editor. After a short introduction on how using the interface, we asked them to design a pedagogical scenario for a real training session to test the feasibility of our methodology. They design together the pedagogical scenario of a training session on "the risks related to population ageing". We present here the scenario of this design session to explain how the design assistance tool system works for supporting the user task. 
The trainers decide to define first the pedagogical strategy. They select the pedagogical element "Strategy". The system collects this element (step 1 of the assistance process). The system identifies the current task "create strategy" and it adds it to the pedagogical design scheme model. A new strategy is added in the pedagogical context of the session context (step 2). The system selects the set of rules for the task "Create a new element" and contextualizes it. For each rule of the set, the system changes the default type of pedagogical element by "Strategy" (step 3). The system tries to apply contextualized rules but no condition on a rule is validated (step 4).

Then trainers decide to add a new element to the scenario instead of reusing an existing one. The system collects the user action (step 1). The assistance system identifies the current activity as "Start new element: from: strategy" and adds the activity to the pedagogical design scheme model (step 2). The system selects the set of rules for the task "Start new element" and contextualizes it (step 3). The system is looking for rules to apply. Two rules are validated and applied. The propriety "context" of the element is initialized to the value of the "context of the scenario" (consistency ensuring). An assistance guide exists to describe the best practices to "create a new element" (step 4 ). The context information is filled and the guide appears (guiding) (Fig 4).

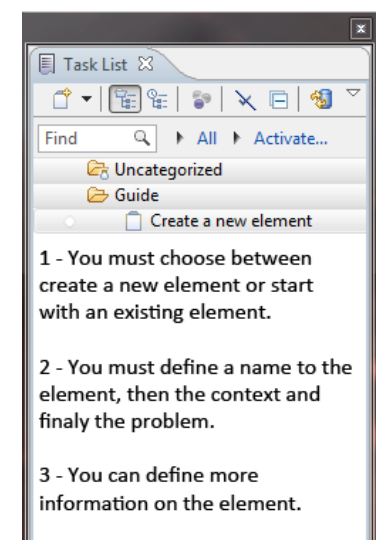

Figure 4: Guide appears on the left on the screen

Trainers define the name of the strategy "raise general awareness of the ageing". The system collects the name (step 1) and identifies the current activity as "Define Name: from: strategy" (step 2). The system selects the set of rules associated to "Define Name" and contextualizes it (step 3). The system tries to apply the set of rules. Only one rule has the condition to be validated and applied: the same name for the same type of pedagogical element was used in a previous design session associated to an objective (step 4). This objective and the link between the objective and the strategy are created and the system informs the user with a message box (action anticipating).

At anytime, the user can change the assistance level to reduce the number of messages or deactivate the assistance system.

\section{DISCUSSION AND CONCLUSIONS}

We have been approached by PARTAGE, an association in charge with back-to work programs, over helping them to improve their learning design activities. Some trainers were involved with us in an iterative participatory design research approach. We have elaborated in a first time a design approach based on patterns and co-constructed with the trainers some patterns which embodie their teaching know-how. In a second time, we have studied with them the assistance process and proposed a context-awareness system to support the design activity, based on pedagogical design schemes and task models. At the end of the pilot study, trainers have reused existing patterns and designed new ones, describing a pedagogical scenario of a learning situation.

The design assistance approach we have proposed is based on a pattern design methodology and has been elaborated by studying a community of trainers involved in specific training needs. In such an approach, patterns are specific to the community and indeed it was our main goal: to embody the know-how of a community of practice in order to allow the sharing and the reuse inside it. Moreover, by allowing both the reuse and the creation of patterns during the design of a learning scenario, we focus the design by the reuse and for the reuse, strengthening by the way the designer's awareness of membership of a community of practice.

To facilitate the design and with the aim of enhancing the reuse, we have proposed a design assistance process which could be considered as an epiphyte system (Giroux, 1996), that is to say the assistance process is implemented independently of the design one, but synchronized with each step of it, by the help of handlers on interaction events. These events are basic and generic primitive design actions (as "create", "modify", "add", etc.) and the set of rules we have defined could be instantiated on any set of pedagogical elements, 
even if we think that the pedagogical elements we have defined here are very standardized (activity, learning objective, role, etc.). Indeed, we improve our approach with another community of practice, computer sciences teachers in our university.

We claim this design and assistance approach we have proposed could be applied with benefits when one wants to (1) capitalize the know-how of an existing teaching community of practice, (2) reinforce the identity of such a community, (3) enhance the productivity of its members, and/or improve the quality of learning designs.

For this, we continue to realize pilot studies on design sessions with PARTAGE and to improve the set of existing patterns and the assistance process; we have started a study with another community in our university, focusing here on the reuse of the assistance process; and we need also to collaborate with other learning communities to improve the assistance rule-based system on another set of pedagogical elements, enlarge the set of pedagogical design schemes we have identified, and better take into account the evolution of the experience of the designer.

\section{REFERENCES}

Amato G., Staraccia U., 1999, User profile modelling and applications to digital librairies, 3rd European Conference on Research and avanced technology for digital libraries , p. 184-187

Andresen K., Gronau N., 2005, Seeking Optimal IT Strategies by the Determination of Adaptability in Domain-Specific Software Applications, IRM 2005.

Bardram J. E., 2005, Activity-Based Support for Mobility and Collaboration in Ubiquitous Computing, International Conference on Ubiquitous Computing.

Clayer JP, Toffolon C, Choquet C,Patterns, 2013, Pedagogical Design Schemes and Process for Instructional Design : With a Pattern-based and Teacher-centered Approach, ICALT.

Dey A.K., Abowd G.D., 2000, Towards a better understanding of context and context-awareness, Conference on Human Factors in Computing System

Dufresne A., Basque J., Paquette G., Leonard M., Lundgren-Cayrol K.,Prom Tep S., 2003, Vers un modèle générique d'assistance aux acteurs $d u$ téléapprentissage . STICEF, v.10

El Kechaï H, Choquet C., 2006 Understanding the Collective Design Process by Analyzing Intermediary Objects, ICALT'2006, p.1047-1051

Emin V., 2010, thesis, Modélisation dirigée par les intentions pour la conception, le partage et la réutilisation de scénarios pédagogiques intégrant les technologies numériques. Joseph Fourier University

Giroux S., Paquette G, Girard J, 1996: Epiphyte Advisor Systems for Collaborative Learning. CALISCE.

Goodyear P., 2005. Educational design and networked learning: Patterns, pattern language and design practice. Australasian Journal of Education Technology, 21(1), 82-101.

Hernández-Leo D, Villasclaras-Fernández E. D., Asensio-Pérez J. I, Dimitriadis Y., Jorrín-Abellán I. M., Ruiz-Requies I., \& Rubia-Avi B., 2006. COLLAGE: A collaborative Learning Design editor based on patterns. Educational Technology \& Society, 9 (1), 58-71

Koper R. ,Olivier B., 2004, In Educational Technology \& Society, 7 (3), Representing the Learning Design of Units of Learning, pp 97-111

Krogmann K., Becker S., 2007, A Case Study on ModelDriven and Conventional Software Development: The Palladio Editor, Software Engineering, ISBN 978-3-88579-200-0

Laurillard D, 2012, Teaching as a Design Science, Building Pedagogical Patterns for Learning and Technology, Routledge, ISBN 978-0-415-8038.

Lopisteguy P, Rieu D, Roose P, 2012, Situation of adaptation, I.S.B.N.: 9782364930292

Mor Y., Winters N, 2007, Design approaches in technology enhanced learning, Journal of Interactive Media in Education

Pangoli S., Paternò F., 1995, In ACM Press, Automatic Generation of Task-oriented Help, Proceedings UIST'95, pp. 181-187.

Paternò F, Alfieri V, 2001, In Handbook of Software Engineering \& Knowledge Engineering, Task Models in Interactive Software Systems

Paquette G., 2004, Instructional engineering for learning objects repositories networks, ICBL 2004, pp 25-36,

Razmerita L., Angehrn A., Nabeth T., 2003, In User Modeling, On the Role of User Models and User Modeling in Knowledge Management Systems, UM 2003

Rech J., Ras E., and Decker B., 2007, Intelligent assistance in german software development: A survey. IEEE Softw.

Rohse S., Anderson T, 2006, Design Patterns for Complex learning, Journal of Learning Design.

Steinberg D., Budinsky F., Paternostro M., Merks E., 2008, EMF: Eclipse Modelling Framework, 2nd edn. Eclipse Series. Addison-Wesley Professional

Schuler D., Namioka A., 1993. Participatory Design: Perspectives on Systems Design, Routledge.

Villiot-Leclercq E., 2007. Modèle de soutien pour l'élaboration et la réutilisation de scénarios pédagogiques, thesis, Université Joseph Fourier/Université de Montréal. 